\title{
Syndromic Surveillance - Reports of Successes from the Field
}

\author{
Antheny Wilson*1, Teresa Hamby², Wei Hou ${ }^{3}$, David J. Swenson ${ }^{4}, K_{r y s t a l}$ Collier ${ }^{5}$ and \\ Michele Hoover ${ }^{1}$
}

'Division of Health Informatics and Surveillance, Centers for Disease Control, Atlanta, GA, USA; ${ }^{2}$ New Jersey Department of Health, Trenton, NJ, USA; ${ }^{3}$ Utah Department of Health, Salt Lake City, UT, USA; ${ }^{4} \mathrm{NH}$ Department of Health, Concord, NH, USA; ${ }^{5}$ Arizona Department of Health Services, Phoenix, AZ, USA

\section{Objective}

This panel will:

- Discuss the importance of identifying and developing success stories

- Highlight successes from state and local health departments to show how syndromic surveillance activities enhance situational awareness and address public health concerns

- Encourage discussion on how to further efforts for developing and disseminating success stories

\section{Introduction}

Syndromic surveillance uses near-real-time emergency department and other health care data for enhancing public health situational awareness and informing public health activities. In recent years, continued progress has been made in developing and strengthening syndromic surveillance activities. At the national level, syndromic surveillance activities are facilitated by the National Syndromic Surveillance Program (NSSP), a collaboration among state and local health departments, the CDC, other federal organizations, and other organizations that enabled collection of syndromic surveillance data in a timely manner, application of advanced data monitoring and analysis techniques, and sharing of best practices. This panel will highlight the importance of success stories. Examples of successes from state and local health departments will be presented and the audience will be encouraged to provide feedback.

\section{Keywords}

success stories; syndromic surveillance; state health departments

\section{Acknowledgments}

Centers for Disease Control, New Hampshire Department of Health, New Jersey Department of Health, Arizona Department of Health Services

\section{${ }^{*}$ Antheny Wilson}

E-mail: nnp9@cdc.gov 